\title{
A Theoretical Investigation of Distortionless Enhancement by Polarization Transfer and Subspectral Editing with a Multiple Quantum Trap NMR Spectroscopy for $\mathbf{C X}_{n}$ Groups
}

\author{
Ö. TEZEL
}

Department of Physics, Faculty of Education

Osmangazi University, Eskişehir, Turkey

\section{A. GenÇTen}

Department of Physics, Faculty of Arts and Sciences

Ondokuz Mayıs University, Samsun, Turkey

A. TokATli, M. ŞAHì AND S. BAHÇELI

Department of Physics, Faculty of Arts and Sciences

Süleyman Demirel University, Isparta, Turkey

(Received September 8, 2003)

Product operator theory is widely used for analytical description of multiple pulse nuclear magnetic resonance experiments for weakly coupled spin systems. Distortionless enhancement by polarization transfer and subspectral editing with a multiple quantum trap NMR experiments are used for spectral assignments of ${ }^{13} \mathrm{C}$ NMR spectra in $\mathrm{CH}_{n}$ groups. First, in this study we proposed and showed theoretically that distortionless enhancement by polarization transfer and subspectral editing with a multiple quantum trap experiments can also be used for subspectral editing of ${ }^{13} \mathrm{C}$ NMR spectra when ${ }^{13} \mathrm{C}$ nuclei coupled to spin- $3 / 2$ nuclei. The product operator technique is applied for the analytical description of ${ }^{13} \mathrm{C}$ distortionless enhancement by polarization transfer and subspectral editing with a multiple quantum trap NMR spectroscopy for ${ }^{13} \mathrm{CX}_{n}\left(I_{\mathrm{X}}=3 / 2 ; n=1,2,3\right)$ groups.

PACS numbers: 82.56.Dj, 82.56.Jn 


\section{Introduction}

One of the major problems of the multipulse NMR spectroscopy has become the determination of proton multiplicities in ${ }^{13} \mathrm{C}$ for the past over two decades. Distortionless enhancement by polarization transfer (DEPT) NMR spectroscopy is used to edit ${ }^{13} \mathrm{C}$ spectra into subspectra containing only $\mathrm{CH}, \mathrm{CH}_{2}$, and $\mathrm{CH}_{3}$ groups while subspectral editing of $\mathrm{CH}_{3}, \mathrm{CH}_{2}, \mathrm{CH}$, and also $\mathrm{C}$ groups can be achieved by using subspectral editing with a multiple quantum trap (SEMUT) NMR spectroscopy [1-4]. As NMR is a quantum mechanical phenomenon, the product operator theory as a quantum mechanical method is widely used for analytical description of multipulse NMR experiments on weakly coupled spin systems in liquids [5-9]. Product operator formalism has been used mostly in two spin-1/2 systems and in systems having spin-1/2 and spin-1 nuclei [9-13]. By using product operator theory, SEMUT NMR spectroscopy is also applied to $\mathrm{CD}_{n}$ groups [13]. Experimental and theoretical investigation of ${ }^{13} \mathrm{C}$ DEPT NMR spectra on $\mathrm{CD}_{n}$ systems is presented elsewhere [14]. Product operator theory for spin-3/2 and its application for 2D $J$-resolved and $3 \mathrm{D} J$-resolved NMR spectroscopies are recently reported $[15,16]$.

In this study, the theoretical investigation on the spectral editing techniques of both DEPT and SEMUT NMR spectroscopy for ${ }^{13} \mathrm{C}$ nuclei coupled to spin-3/2 nuclei are separately presented. For the first time, this will be an application of product operator theory to DEPT and SEMUT NMR spectroscopy for ${ }^{13} \mathrm{CX}_{n}\left(I_{\mathrm{X}}=3 / 2, n=1,2,3\right)$ groups. For example, $\mathrm{X}$ can be ${ }^{11} \mathrm{~B}$ nuclei $(I=3 / 2 ; 80.1 \%)$ in boron compounds.

\section{Theory}

The product operator theory is the expression of the density matrix operator in terms of matrix representation of angular momentum operators for individual spins. Time dependence of the density matrix is given by $[6,7]$ :

$$
\sigma(t)=\exp (-\mathrm{i} H t) \sigma(0) \exp (\mathrm{i} H t)
$$

where $H$ is the total Hamiltonian which consists of radio frequency (rf) pulse, chemical shift and spin-spin coupling Hamiltonians and $\sigma(0)$ is the density matrix at $t=0$. After employing the Hausdorff formula [7],

$$
\begin{aligned}
\exp (-\mathrm{i} H t) A \exp (\mathrm{i} H t)=A-(\mathrm{i} t)[H, A]+\frac{(\mathrm{i} t)^{2}}{2 !}[H,[H, A]] \\
\quad-\frac{(\mathrm{i} t)^{3}}{3 !}[H,[H,[H, A]]]+\ldots,
\end{aligned}
$$

the evolutions of product operators under the rf pulse, chemical shift, and spin-spin coupling Hamiltonians can easily be obtained [5-7]. The details on the evolution of product operators under these Hamiltonians and the short hand notations can be found elsewhere [5-8]. For $I S_{n}(I=1 / 2, S=3 / 2)$ spin system, by using the 
Hausdorff formula, evolutions of $I_{x}, I_{y}, I_{x} S_{z}$, and $I_{y} S_{z}$ product operator under the spin-spin coupling Hamiltonian $H_{J}=2 \pi J I_{z} S_{z}$ can be obtained as follows [15]:

$$
\begin{aligned}
& I_{x} \stackrel{2 \pi J I_{z} S_{z} t}{\longrightarrow} I_{x} E_{S}\left( \pm \frac{3}{2}\right) \cos (3 \pi J t)+\frac{2}{3} I_{y} S_{z} E_{S}\left( \pm \frac{3}{2}\right) \sin (3 \pi J t) \\
&+I_{x} E_{S}\left( \pm \frac{1}{2}\right) \cos (\pi J t)+2 I_{y} S_{z} E_{S}\left( \pm \frac{1}{2}\right) \sin (\pi J t) \\
& I_{y} \stackrel{2 \pi J I_{z} S_{z} t}{\longrightarrow} I_{y} E_{S}\left( \pm \frac{3}{2}\right) \cos (3 \pi J t)-\frac{2}{3} I_{x} S_{z} E_{S}\left( \pm \frac{3}{2}\right) \sin (3 \pi J t) \\
& \quad+I_{y} E_{S}\left( \pm \frac{1}{2}\right) \cos (\pi J t)-2 I_{x} S_{z} E_{S}\left( \pm \frac{1}{2}\right) \sin (\pi J t) \\
& I_{x} S_{z} \stackrel{2 \pi J I_{z} S_{z} t}{\longrightarrow} I_{x} S_{z} E_{S}\left( \pm \frac{3}{2}\right) \cos (3 \pi J t)+\frac{3}{2} I_{y} E_{S}\left( \pm \frac{3}{2}\right) \sin (3 \pi J t) \\
& \quad+I_{x} S_{z} E_{S}\left( \pm \frac{1}{2}\right) \cos (\pi J t)+\frac{1}{2} I_{y} E_{S}\left( \pm \frac{1}{2}\right) \sin (\pi J t) \\
& I_{y} S_{z} \stackrel{2 \pi J I_{z} S_{z} t}{\longrightarrow} I_{y} S_{z} E_{S}\left( \pm \frac{3}{2}\right) \cos (3 \pi J t)-\frac{3}{2} I_{x} E_{S}\left( \pm \frac{3}{2}\right) \sin (3 \pi J t) \\
& \quad+I_{y} S_{z} E_{S}\left( \pm \frac{1}{2}\right) \cos (\pi J t)-\frac{1}{2} I_{x} E_{S}\left( \pm \frac{1}{2}\right) \sin (\pi J t),
\end{aligned}
$$

where $E_{S}$ is the identity operator for $S$ nucleus. Recently, these results were first obtained and then applied to analytical description of heteronuclear $2 \mathrm{D} J$-resolved NMR spectroscopy for $I S_{n}(I=1 / 2, S=3 / 2)$ spin systems [15].

\section{DEPT and SEMUT NMR spectroscopies for ${ }^{13} \mathbf{C X}_{n}\left(I_{\mathrm{X}}=3 / 2 ; n=1,2,3\right)$ groups}

In this section of study, by using the product operator theory, analytical description of DEPT and SEMUT NMR spectroscopies for ${ }^{13} \mathrm{CX}_{n}\left(I_{\mathrm{X}}=3 / 2\right)$ groups will be presented. The pulse sequences employed for DEPT and SEMUT NMR spectroscopies are illustrated in Fig. 1 and Fig. 2, respectively. As seen in these figures, the density matrix operators at each stage of the experiment are labeled with the numbers. Furthermore, in our calculations $I$ and $S$ have been treated as ${ }^{13} \mathrm{C}$ and $\mathrm{X}$ nuclei, respectively.

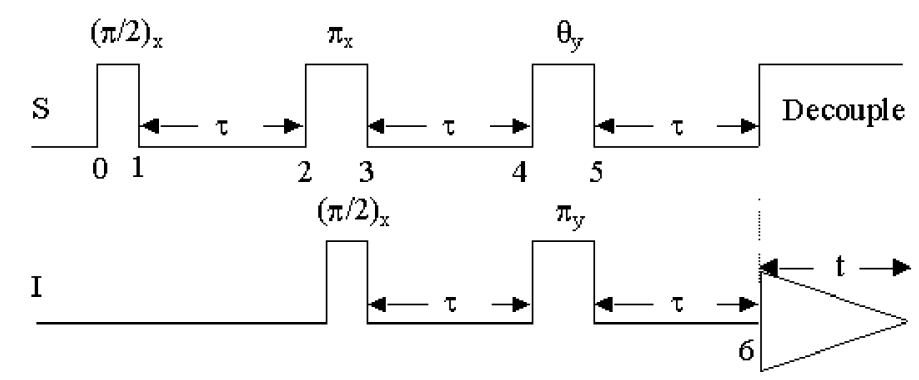

Fig. 1. The pulse sequence of DEPT NMR spectroscopy. In this pulse sequence, the optimum value of $\tau$ is $(2 J)^{-1}$ for $I S_{n}(I=1 / 2, S=3 / 2)$ spin system. 


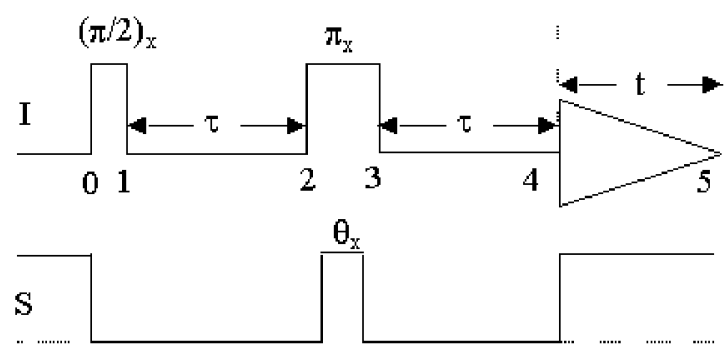

Fig. 2. The pulse sequence of SEMUT NMR spectroscopy. In this pulse sequence, the optimum value of $\tau$ is $(2 J)^{-1}$ for $I S_{n}(I=1 / 2, S=3 / 2)$ spin system. During $t$ only the chemical shift evolution takes place.

\subsection{DEPT NMR}

For $I S(\mathrm{CX})$ spin system, $\sigma_{0}=S_{z}$ is the density matrix operator at thermal equilibrium in DEPT NMR. By using the pulse sequence in Fig. 1, the following density matrices for each labeled point are obtained:

$$
\begin{aligned}
& \sigma_{1}=-S_{y}, \\
& \sigma_{2}=2 I_{z} S_{x}, \\
& \sigma_{3}=\sigma_{4}=-2 I_{y} S_{x}, \\
& \sigma_{5}=2 I_{y} S_{z} \sin \theta, \\
& \sigma_{6}=\left[3 I_{x} E_{S}\left( \pm \frac{3}{2}\right)-I_{x} E_{S}\left( \pm \frac{1}{2}\right)\right] S_{\theta},
\end{aligned}
$$

where $S_{n \theta}=\sin (n \theta)$. It is assumed that during $\tau$, the relaxation and evolution under chemical shift do not exist. The chemical shift evolution takes place only during detection time, $t$. The density operator at point 6 in Fig. 1 implies that the calculation can be stopped at this point because of the decoupling between $I$ and $S$ nuclei after that. When the signal is detected only from $x$ axis, the magnetization along $x$ axis is proportional to $\left\langle I_{x}\right\rangle$ and becomes

$$
M_{x} \alpha\left\langle I_{x}\right\rangle=\operatorname{Tr}\left(I_{x} \sigma_{6}\right) .
$$

For $I S(I=1 / 2, S=3 / 2)$ spin system by considering the following calculated trace values $[15]$ :

$$
\operatorname{Tr}\left[I_{x} I_{x} E_{S}\left( \pm \frac{3}{2}\right)\right]=\operatorname{Tr}\left[I_{x} I_{x} E_{S}\left( \pm \frac{1}{2}\right)\right]=\frac{1}{2} \operatorname{Tr}\left(I_{x} I_{x}\right)=1
$$

we obtain

$$
\left\langle I_{x}\right\rangle(I S)=2 S_{\theta} .
$$

For $I S_{2}$ spin system 


$$
\sigma_{0}=S_{1 z}+S_{2 z}
$$

and by using the same pulse sequence, we have

$$
\begin{aligned}
& \sigma_{1}=-\left(S_{1 y}+S_{2 y}\right) \\
& \sigma_{2}=2 I_{z} S_{1 x}+2 I_{z} S_{2 x} \\
& \sigma_{3}=-2\left[\begin{array}{c}
I_{y} E_{S}\left( \pm \frac{3}{2}, \pm \frac{3}{2}\right)+I_{y} E_{S}\left( \pm \frac{3}{2}, \pm \frac{1}{2}\right) \\
+I_{y} E_{S}\left( \pm \frac{1}{2}, \pm \frac{3}{2}\right)+I_{y} E_{S}\left( \pm \frac{1}{2}, \pm \frac{1}{2}\right)
\end{array}\right]\left(S_{1 x}+S_{2 x}\right)
\end{aligned}
$$

and

$$
\sigma_{6}=I_{x}\left[\begin{array}{c}
3 E_{S}\left( \pm \frac{3}{2}, \pm \frac{3}{2}\right)+3 E_{S}\left( \pm \frac{3}{2}, \pm \frac{1}{2}\right) \\
-E_{S}\left( \pm \frac{1}{2}, \pm \frac{3}{2}\right)-E_{S}\left( \pm \frac{1}{2}, \pm \frac{1}{2}\right)
\end{array}\right] S_{2 \theta}
$$

By using the values [15]:

$$
\begin{aligned}
& \operatorname{Tr}\left[I_{x} I_{x} E_{S}\left( \pm \frac{3}{2}, \pm \frac{3}{2}\right)\right]=\operatorname{Tr}\left[I_{x} I_{x} E_{S}\left( \pm \frac{3}{2}, \pm \frac{1}{2}\right)\right]=\operatorname{Tr}\left[I_{x} I_{x} E_{S}\left( \pm \frac{1}{2}, \pm \frac{3}{2}\right)\right] \\
& \quad=\operatorname{Tr}\left[I_{x} I_{x} E_{S}\left( \pm \frac{1}{2}, \pm \frac{1}{2}\right)\right]=\frac{1}{4} \operatorname{Tr}\left(I_{x} I_{x}\right)=2 \\
& \left\langle I_{x}\right\rangle\left(I S_{2}\right)=8 S_{2 \theta}
\end{aligned}
$$

is found.

In the case of $I S_{3}$ spin system

$$
\sigma_{0}=S_{1 z}+S_{2 z}+S_{3 z}
$$

Using the same pulse sequence, the following density matrices can be obtained:

$$
\begin{aligned}
& \sigma_{1}=-\left(S_{1 y}+S_{2 y}+S_{3 y}\right) \\
& \sigma_{2}=2 I_{z} S_{1 x}+2 I_{z} S_{2 x}+2 I_{z} S_{3 x} \\
& \sigma_{3}=-2 I_{y}\left[\begin{array}{c}
E_{S}\left( \pm \frac{3}{2}, \pm \frac{3}{2}, \pm \frac{3}{2}\right)+E_{S}\left( \pm \frac{3}{2}, \pm \frac{3}{2}, \pm \frac{1}{2}\right) \\
+E_{S}\left( \pm \frac{3}{2}, \pm \frac{1}{2}, \pm \frac{3}{2}\right)+E_{S}\left( \pm \frac{3}{2}, \pm \frac{1}{2}, \pm \frac{1}{2}\right) \\
+E_{S}\left( \pm \frac{1}{2}, \pm \frac{3}{2}, \pm \frac{3}{2}\right)+E_{S}\left( \pm \frac{1}{2}, \pm \frac{3}{2}, \pm \frac{1}{2}\right) \\
+E_{S}\left( \pm \frac{1}{2}, \pm \frac{1}{2}, \pm \frac{3}{2}\right)+E_{S}\left( \pm \frac{1}{2}, \pm \frac{1}{2}, \pm \frac{1}{2}\right)
\end{array}\right] \\
& \quad \times\left(S_{1 x}+S_{2 x}+S_{3 x}\right) .
\end{aligned}
$$

Then, we have

$$
\sigma_{6}=I_{x}\left[\begin{array}{c}
9 E_{S}\left( \pm \frac{3}{2}, \pm \frac{3}{2}, \pm \frac{3}{2}\right)+5 E_{S}\left( \pm \frac{3}{2}, \pm \frac{3}{2}, \pm \frac{1}{2}\right) \\
+5 E_{S}\left( \pm \frac{3}{2}, \pm \frac{1}{2}, \pm \frac{3}{2}\right)+E_{S}\left( \pm \frac{3}{2}, \pm \frac{1}{2}, \pm \frac{1}{2}\right) \\
+5 E_{S}\left( \pm \frac{1}{2}, \pm \frac{3}{2}, \pm \frac{3}{2}\right)+E_{S}\left( \pm \frac{1}{2}, \pm \frac{3}{2}, \pm \frac{1}{2}\right) \\
+E_{S}\left( \pm \frac{1}{2}, \pm \frac{1}{2}, \pm \frac{3}{2}\right)-3 E_{S}\left( \pm \frac{1}{2}, \pm \frac{1}{2}, \pm \frac{1}{2}\right)
\end{array}\right] S_{\theta} C_{\theta}^{2},
$$

where $C_{\theta}=\cos \theta$. And by using the values [15]: 


$$
\begin{aligned}
\operatorname{Tr}[ & \left.I_{x} I_{x} E_{S}\left( \pm \frac{3}{2}, \pm \frac{3}{2}, \pm \frac{3}{2}\right)\right]=\operatorname{Tr}\left[I_{x} I_{x} E_{S}\left( \pm \frac{3}{2}, \pm \frac{3}{2}, \pm \frac{1}{2}\right)\right] \\
& =\operatorname{Tr}\left[I_{x} I_{x} E_{S}\left( \pm \frac{3}{2}, \pm \frac{1}{2}, \pm \frac{3}{2}\right)\right]=\operatorname{Tr}\left[I_{x} I_{x} E_{S}\left( \pm \frac{1}{2}, \pm \frac{3}{2}, \pm \frac{3}{2}\right)\right] \\
& =\operatorname{Tr}\left[I_{x} I_{x} E_{S}\left( \pm \frac{3}{2}, \pm \frac{1}{2}, \pm \frac{1}{2}\right)\right]=\operatorname{Tr}\left[I_{x} I_{x} E_{S}\left( \pm \frac{1}{2}, \pm \frac{3}{2}, \pm \frac{1}{2}\right)\right] \\
& =\operatorname{Tr}\left[I_{x} I_{x} E_{S}\left( \pm \frac{1}{2}, \pm \frac{1}{2}, \pm \frac{3}{2}\right)\right]=\operatorname{Tr}\left[I_{x} I_{x} E_{S}\left( \pm \frac{1}{2}, \pm \frac{1}{2}, \pm \frac{1}{2}\right)\right] \\
& =\frac{1}{8} \operatorname{Tr}\left(I_{x} I_{x}\right)=4 \\
\left\langle I_{x}\right\rangle & \left(I S_{3}\right)=24\left(S_{\theta}+S_{3 \theta}\right)
\end{aligned}
$$

is obtained.

\subsection{SEMUT NMR}

For the analytical description of SEMUT NMR spectroscopy for ${ }^{13} \mathrm{CX}_{n}$ ( $\left.I_{\mathrm{X}}=3 / 2, n=1,2,3\right)$ groups ( $I S_{n}$ spin systems), the pulse sequence in Fig. 2 is used. The optimum value for $\tau$ is $(2 J)^{-1}$. For $I S(I=1 / 2, S=3 / 2)$ spin system $\sigma_{0}=I_{z}$ and the following density matrices for each labeled point are obtained:

$$
\begin{aligned}
& \sigma_{1}=-I_{y} \\
& \sigma_{2}=-\frac{2}{3} I_{x} S_{z} E_{S}\left( \pm \frac{3}{2}\right)+2 I_{x} S_{z} E_{S}\left( \pm \frac{1}{2}\right) \\
& \sigma_{3}=I_{x} S_{z}\left[-\frac{2}{3} E_{S}\left( \pm \frac{3}{2}\right)+2 E_{S}\left( \pm \frac{1}{2}\right)\right] C_{\theta} \\
& \sigma_{4}=I_{y}\left[E_{S}\left( \pm \frac{3}{2}\right)+E_{S}\left( \pm \frac{1}{2}\right)\right] C_{\theta} .
\end{aligned}
$$

When the signal is detected only from $y$ axis, the magnetization along $y$ axis is proportional to $\left\langle I_{y}\right\rangle$ and becomes

$$
M_{y} \alpha\left\langle I_{y}\right\rangle=\operatorname{Tr}\left(I_{y} \sigma_{4}\right)
$$

Therefore, we obtain

$$
\left\langle I_{y}\right\rangle(I S)=2 C_{\theta} .
$$

For $I S_{2}$ spin system, the following density matrices are found:

$$
\begin{aligned}
& \sigma_{1}=-I_{y} \\
& \sigma_{2}=\left[\begin{array}{c}
\frac{4}{9} I_{y} E_{S}\left( \pm \frac{3}{2}, \pm \frac{3}{2}\right)-\frac{4}{3} I_{y} E_{S}\left( \pm \frac{3}{2}, \pm \frac{1}{2}\right) \\
-\frac{4}{3} I_{y} E_{S}\left( \pm \frac{1}{2}, \pm \frac{3}{2}\right)+4 I_{y} E_{S}\left( \pm \frac{1}{2}, \pm \frac{1}{2}\right)
\end{array}\right]\left(S_{1 z} S_{2 z}\right) \\
& \sigma_{4}=I_{y}\left[\begin{array}{c}
E_{S}\left( \pm \frac{3}{2}, \pm \frac{3}{2}\right)+E_{S}\left( \pm \frac{3}{2}, \pm \frac{1}{2}\right) \\
+E_{S}\left( \pm \frac{1}{2}, \pm \frac{3}{2}\right)+E_{S}\left( \pm \frac{1}{2}, \pm \frac{1}{2}\right)
\end{array}\right] C_{\theta}^{2}
\end{aligned}
$$

Then,

$$
\left\langle I_{y}\right\rangle\left(I S_{2}\right)=8 C_{\theta}^{2} .
$$


For $I S_{3}$ spin system

$$
\sigma_{4}=I_{y}\left[\begin{array}{c}
E_{S}\left( \pm \frac{3}{2}, \pm \frac{3}{2}, \pm \frac{3}{2}\right)+E_{S}\left( \pm \frac{3}{2}, \pm \frac{3}{2}, \pm \frac{1}{2}\right) \\
+E_{S}\left( \pm \frac{3}{2}, \pm \frac{1}{2}, \pm \frac{3}{2}\right)+E_{S}\left( \pm \frac{3}{2}, \pm \frac{1}{2}, \pm \frac{1}{2}\right) \\
+E_{S}\left( \pm \frac{1}{2}, \pm \frac{3}{2}, \pm \frac{3}{2}\right)+E_{S}\left( \pm \frac{1}{2}, \pm \frac{3}{2}, \pm \frac{1}{2}\right) \\
+E_{S}\left( \pm \frac{1}{2}, \pm \frac{1}{2}, \pm \frac{3}{2}\right)+E_{S}\left( \pm \frac{1}{2}, \pm \frac{1}{2}, \pm \frac{1}{2}\right)
\end{array}\right] C_{\theta}^{3}
$$

Then,

$$
\left\langle I_{y}\right\rangle\left(I S_{3}\right)=32 C_{\theta}^{3}
$$

\section{Discussion}

$\operatorname{Tr}\left(I_{x} \sigma_{6}\right)=\operatorname{Tr}\left(I_{x} \sigma_{f}\right)$ and $\operatorname{Tr}\left(I_{y} \sigma_{4}\right)=\operatorname{Tr}\left(I_{y} \sigma_{f}\right)$ values obtained in the preceding section for $I S_{n}(I=1 / 2, S=3 / 2 ; n=1,2,3)$ spin systems for both DEPT and SEMUT NMR spectroscopies represent the free induction decay (FID) signals, respectively. These values can be normalized by multiplication with $4 / \operatorname{Tr}(E)$. Here $E$ is the unity product operator for corresponding spin system. Obtained $\operatorname{Tr}\left(I_{x} \sigma_{f}\right)$ and $\operatorname{Tr}\left(I_{y} \sigma_{f}\right)$ values and their normalized forms are presented in Table I and Table II for DEPT and SEMUT NMR spectroscopies, respectively, for both $I S_{n}$

TABLE I

$\operatorname{Tr}\left(I_{x} \sigma_{f}\right)$ values and their normalized forms for both $I S_{n}(I=1 / 2$, $S=1 / 2 ; n=1,2,3)$ and $I S_{n}(I=1 / 2, S=3 / 2 ; n=1,2,3)$ spin systems for DEPT NMR spectroscopy.

\begin{tabular}{c|c|c|c|c}
\hline \hline \multirow{2}{*}{ Spin system } & \multicolumn{2}{|c|}{$\operatorname{Tr}\left(I_{x} \sigma_{f}\right)$} & \multicolumn{2}{c}{ Normalized $\operatorname{Tr}\left(I_{x} \sigma_{f}\right)$} \\
\cline { 2 - 5 } & $S=1 / 2^{a}$ & $S=3 / 2^{b}$ & $S=1 / 2^{a}$ & $S=3 / 2^{b}$ \\
\hline$I S$ & $S_{\theta}$ & $2 S_{\theta}$ & $S_{\theta}$ & $S_{\theta}$ \\
$I S_{2}$ & $2 S_{2 \theta}$ & $8 S_{2 \theta}$ & $S_{2 \theta}$ & $S_{2 \theta}$ \\
$I S_{3}$ & $3\left(S_{\theta}+S_{3 \theta}\right)$ & $24\left(S_{\theta}+S_{3 \theta}\right)$ & $\frac{3}{4}\left(S_{\theta}+S_{3 \theta}\right)$ & $\frac{3}{4}\left(S_{\theta}+S_{3 \theta}\right)$ \\
\hline
\end{tabular}

${ }^{a}[6,7],{ }^{b}$ obtained in this study.

TABLE II

$\operatorname{Tr}\left(I_{y} \sigma_{f}\right)$ values and their normalized forms for both $I S_{n}(I=1 / 2, S=1 / 2 ; n=1,2,3)$ and $I S_{n}(I=1 / 2$, $S=3 / 2 ; n=1,2,3)$ spin systems for SEMUT NMR spectroscopy.

\begin{tabular}{c|c|c|c|c}
\hline \hline \multirow{2}{*}{ Spin system } & \multicolumn{2}{|c|}{$\operatorname{Tr}\left(I_{y} \sigma_{f}\right)$} & \multicolumn{2}{c}{ Normalized $\operatorname{Tr}\left(I_{y} \sigma_{f}\right)$} \\
\cline { 2 - 5 } & $S=1 / 2^{a}$ & $S=3 / 2^{b}$ & $S=1 / 2^{a}$ & $S=3 / 2^{b}$ \\
\hline$I S$ & $C_{\theta}$ & $2 C_{\theta}$ & $C_{\theta}$ & $C_{\theta}$ \\
$I S_{2}$ & $2 C_{\theta}^{2}$ & $8 C_{\theta}^{2}$ & $C_{\theta}^{2}$ & $C_{\theta}^{2}$ \\
$I S_{3}$ & $4 C_{\theta}^{3}$ & $32 C_{\theta}^{3}$ & $C_{\theta}^{3}$ & $C_{\theta}^{3}$ \\
\hline
\end{tabular}

${ }^{a}[2],{ }^{b}$ obtained in this study. 
$(I=1 / 2, S=1 / 2 ; n=1,2,3)$ and $I S_{n}(I=1 / 2, S=3 / 2 ; n=1,2,3)$ spin systems. As seen in Table I and Table II normalized forms of $\operatorname{Tr}\left(I_{x} \sigma_{f}\right)$ and $\operatorname{Tr}\left(I_{y} \sigma_{f}\right)$ values are the same for both spin systems in both DEPT and SEMUT NMR experiments. Therefore, DEPT and SEMUT NMR experiments can be also used for ${ }^{13} \mathrm{CX}_{n}\left(I_{\mathrm{X}}=3 / 2 ; n=1,2,3\right)$ groups similar to ${ }^{13} \mathrm{CH}_{n}(n=1,2,3)$ groups. Although, it is well known that $J$ coupling to quadrupolar nuclei cannot be observed because of the existing electric field gradient (EFG). But in some symmetries of molecules, the quadrupolar nuclei have zero or low EFG and $J$ coupling can be observed $[17,18]$. As there exists $J$ coupling in DEPT and SEMUT NMR pulse sequences, SEMUT and DEPT NMR spectroscopy of ${ }^{13} \mathrm{C}$ can be observed in some molecules in which coupled spin-3/2 nuclei have zero or low EFG. As NMR plays an important role in boron chemistry, $\mathrm{X}$ can be ${ }^{11} \mathrm{~B}$ nuclei in ${ }^{13} \mathrm{CX}_{n}\left(I_{\mathrm{X}}=3 / 2 ; n=1,2,3\right)$ groups [19]. For example alkyl boron compound can be a model molecule for proposed DEPT and SEMUT experiments. Hydroboration of alkenes can give theoretically the following compound:

$$
\mathrm{C}\left[\mathrm{B}(\mathrm{Me})_{2}\right]_{3} \mathrm{C}\left[\mathrm{B}(\mathrm{Me})_{2}\right]_{2} \mathrm{C}\left[\mathrm{B}(\mathrm{Me})_{2}\right]_{3} \text {. }
$$

In this compound $\mathrm{CB}, \mathrm{CB}_{2}$, and $\mathrm{CB}_{3}$ groups exist. Although this compound is sterically hindered, this can be synthesized by hydroboration of corresponding alkene derivatives.

\section{Conclusion}

DEPT and SEMUT NMR experiments are widely used for spectral editing of ${ }^{13} \mathrm{C}$ nuclei in $\mathrm{CH}_{n}$ groups. In this study, by using product operator theory, we have proposed and showed theoretically that DEPT and SEMUT NMR experiments can be also applied for subspectral editing of ${ }^{13} \mathrm{C}$ NMR spectra when ${ }^{13} \mathrm{C}$ nuclei are coupled to spin-3/2 nuclei. But, one should be aware of the fact that in order to observe DEPT and SEMUT NMR experiments for these spin systems, spin-3/2 nuclei in a molecule must have zero or low EFG.

\section{Acknowledgment}

We would like to thank Dr. Y. Bekdemir (Department of Chemistry, Ondokuz Mayss University, Samsun, Turkey) for his comments and suggestions about hydroboration of alkenes.

\section{References}

[1] D.M. Doddrell, D.T. Pegg, M.R. Bendall, J. Magn. Reson. 48, 323 (1982).

[2] H. Bildsøe, S. Dønstrup, H.J. Jakobsen, O.W. Sørensen, J. Magn. Reson. 53, 154 (1983) 
[3] U.B. Sørensen, H. Bildsøe, H.J. Jakobsen, O.W. Sørensen, J. Magn. Reson. 65, $222(1985)$.

[4] M.R. Bendall, D.T. Pegg, J. Magn. Reson. 59, 237 (1984).

[5] O.W. Sørensen, G.W. Eich, M.H. Levitt, G. Bodenhausen, R.R. Ernst, Prog. Nucl. Magn. Reson. Spectrosc. 16, 163 (1983).

[6] R.R. Ernst, G. Bodenhausen, A. Wokaun, Principles of Nuclear Magnetic Resonance in One and Two Dimensions, Clarendon Press, Oxford 1987.

[7] N. Chandrakumar, S. Subramanian, Modern Techniques in High Resolution FT NMR, Springer, New York 1987.

[8] P.J. Hore, J.A. Jones, S. Wimperis, NMR: The Toolkit, Oxford University Press, New York 2000.

[9] F.J.M. Van de Ven, C.W. Hilbers, J. Magn. Reson. 54, 512 (1983).

[10] N. Chandrakumar, J. Magn. Reson. 60, 28 (1984).

[11] I.S. Podkorytov, Concepts Magn. Reson. 9, 117 (1997).

[12] A. Gençten, T. Özdoğan, F. Köksal, Spect. Lett. 31, 981 (1988).

[13] A. Gençten, Ö. Tezel, A. Köroğlu, Appl. Magn. Reson. 20, 265 (2001).

[14] T.T. Nakashima, R.E.D. McClung, B.K. John, J. Magn. Reson. 58, 27 (1984).

[15] A. Gençten, Ö. Tezel, S. Bahçeli, Chem. Phys. Lett. 351, 109 (2002).

[16] İ. Şaka, Ö. Tezel, A. Gençten, Z. Naturforsch. A 58, 139 (2003).

[17] J.C.P. Sanders, G.J. Schrobilgin, in: Multinuclear Magnetic Resonance in Liquids and Solids - Chemical Applications, Eds. P. Granger, R.K. Harris, Kluwer Academic, Dordrecht 1990, p. 157.

[18] J.W. Akitt, W.S. McDonald, J. Magn. Reson. 58, 401 (1984).

[19] D. Reed, Chem. Soc. Rev. 22, 109 (1993). 